\title{
NMR Spectral Studies of Some Six-membered and Seven-membered Saturated Heterocyclic Compounds and Preparation of Some Six-membered Saturated Heterocyclic Compounds and NMR Measurements
}

\author{
G. Mathubala and N. Babitha
}

Bharath University, Selaiyur, Chennai-600 073, India madhu2705@gmail.com,n_babitha@rediffmail.com

\begin{abstract}
(1)N-Nitroso-t[3]-methyl-r[2],c[6]-diphenyl piperidin-4-one was prepared; the procedure of Noller and Baliah was followed to prepare the compound [1].

By taking solution of the compound in a suitable solvent, NMR measurements were taken.
\end{abstract}

Keywords: Ammonium Acetate, Ammonia, Anhydrous, Benzaldehyde, Crush, Drop-wise, Extract, Melting Point, Petroleum Ether, Stirring

Preparation of Compounds

$\mathrm{N}$ - Nitroso - $\mathrm{t}[3]$ - methyl -r[2],c[6]-diphenylpiperidin - 4 - one [59]

\section{Introduction}

The procedure adopted was that of Noller and Baliah 48. Dry ammonium acetate $[0.1 \mathrm{~mol}]$ was dissolved in glacial acetic acid $[15 \mathrm{ml}]$ and the solution was mixed with benzaldehyde $[0.2 \mathrm{~mol}]$ and butanone $[0.1 \mathrm{~mol}]$. The mixture was just heated to boil and allowed to stand at room temperature overnight. Concentrated hydrochloric acid [15 ml] was then added and the precipitated hydrochloride was collected, washed with ethanol - ether [1:1] and crystallised from ethanol - ether [1:5]. The compound melted at $224^{\circ} \mathrm{C}$ with decomposition. A suspension of the hydrochloride in acetone was treated with strong ammonia, and the free base was obtained by dilution with water. The compound $\mathrm{t}[3]$ - methyl -r[2],c[6]- diphenylpiperidin-4one, after crystallisation from ethanol, melted at $860^{\circ} \mathrm{C}$ [li. Mp. $86-87^{\circ} \mathrm{C}$ ]

A mixture of $t[3]$ - methyl $-r[2], c[6]-$ diphenylpiperidin-4-one $[0.80 \mathrm{~g}, 3.02 \mathrm{~m} . \mathrm{mol}]$ and concentrated hydrochloric acid [0.4 ml] was dissolved in a 1:1 ethanol water mixture $[25 \mathrm{ml}]$. The temperature of the solution was kept at $65-70^{\circ} \mathrm{C}$, and while stirring, a solution of sodium nitrate $[0.21 \mathrm{~g}, 3.0 \mathrm{~m} . \mathrm{mol}]$ in a $1: 1$ ethanol-water mixture [15 ml] was added drop wise over a period of one hour. The heating and stirring was continued for another two hours. The reaction mixture was extracted 4 times with ether $[100 \mathrm{ml}]$, and the extracts were washed with water several times. The combined ethanol layer was dried over anhydrous $\mathrm{NaSO}_{4}$. After removal of ether, the crude product was recrystallised twice from ethanol to give colourless spongy solid. The melting point is $124-125^{\circ} \mathrm{C}$.

\section{2. ${ }^{13} \mathrm{C}$ NMR Spectroscopy}

\section{2. $1{ }^{13} \mathrm{C}$ Chemical Shift}

${ }^{13} \mathrm{C}$ chemical shift depends on many factors. Lambert et al. $^{62}$ have studied the effect of heteroatom in 
monoheteracyclohexanes 26 on the shifts of ring carbons. The $\alpha$ - shift is a steep function of the electronegativity of the heteroatom X. An increase by one unit in electronegativity produces a downfield shift of about $50 \mathrm{ppm}$. However, the effect of hetero atom electronegativity on $\beta$ and $\gamma$ carbons is small.

The effect of introduction of heteroatom in 27 was studied by Berlin et al. ${ }^{1}$ The deshielding effect of heteroatom on the benzylic carbon decreases in the order $\mathrm{O}>$ $\mathrm{NMe}>\mathrm{NH}>\mathrm{S}$. The heteroatom causes an upfield shift in the carbonyl resonance, and this upfield shift has been attributed to a field effect.

The $\gamma$ - effect being a property of at least four atoms has a Torsional component. The dihedral angle between the resonating carbon and perturbing $\gamma$ substituent can range from 0 to $180^{\circ}$. Lambert and Vaganas64 investigated several 3, 3 - dimethyl heteracyclo hexanes 28 for studying $\gamma$ - effects. In 28, C - 4 and the axial 3 - methyl carbon are $\gamma$ gauche effect of $\mathrm{X}$ on $\mathrm{C}-4$ was also found to be linear but with lesser slope in 28 [-3.5 ppm/electronegativity unit] than in 26 [-5.3 ppm/electronegativity unit]. In considerable contrast, the effect of $\mathrm{X}$ on axial 3-methyl carbon was found to be irregular and small.
Berlin et al, ${ }^{1}$ have investigated cis and trans - 2,6 diphenyl -1 - hetera - 4 - cyclohexanones 29 and 30. The chemical shifts for these compounds are listed in Table 1.

The $\alpha$ and $\beta$ carbons of the trans isomer appear markedly upfield suggesting that $\alpha$ and $\beta$ effects are smaller for an axial substituent than for equatorial substituent.

Buchnann 65 noticed upfield shifts in 31a, relative to the equatorial counterpart $31 \mathrm{~b}$ by studying the $13 \mathrm{C}$ NMR Spectrum of Vinylcyclohexane at low temperature. The chemical shifts of $31 \mathrm{a}$ and $31 \mathrm{~b}$ are listed in Table 2.

Authors have investigated the conformational dependence of ${ }^{13} \mathrm{C}$ shielding in molecules possessing heteroatoms gauche and anti to carbon nuclei.

Authors have measured the $\gamma$ - anti effects in 3,3dimethylcyclohexyl derivatives 32 using $C$ [3] and C [5] as the probes. ${ }^{13} \mathrm{C}$ chemical shifts of $\mathrm{C}[3]$ and $\mathrm{C}[5]$ are given in Table 3.

All $\gamma$ - anti substituents cause increased shielding on C [5], and this has been attributed to the presence of $\alpha$ and $\gamma$ protons. The $\alpha \gamma-$ anti effect C [3] is found to be rather deshielding.

The $\gamma$ - gauche effect, arising from the steric compression caused by 1,3-syn-diaxial interaction, has

Table 1. ${ }^{13} \mathrm{C}$ Chemical shifts for $1-$ hetera-2,6-diaryl-4-cyclohexanones

\begin{tabular}{ccccc}
\hline Compound & $\mathrm{X}$ & $\mathrm{C}-\mathbf{2}$ & $\mathrm{C}-\mathbf{3}$ & $\mathrm{C}-\mathbf{4}$ \\
\hline 29a & $\mathrm{S}$ & 48.15 & 50.24 & 206.78 \\
30a & $\mathrm{S}$ & 43.78 & 48.41 & 207.75 \\
29b & O & 78.64 & 49.46 & 205.20 \\
30b & O & 73.27 & 46.19 & 205.87 \\
\hline
\end{tabular}

Table 2. ${ }^{13} \mathrm{C}$ Chemical Shifts of Axial and Equatorial Vinylcyclohexanones

\begin{tabular}{ccccccc}
\hline Compound & $\mathrm{C}-\mathbf{1}$ & $\mathrm{C}-2, \mathrm{C}-\mathbf{6}$ & $\mathrm{C}-\mathbf{3}$ & $\mathrm{C}-\mathbf{4}$ & $\mathrm{C}-\boldsymbol{\alpha}$ & $\mathrm{C}-\boldsymbol{\beta}$ \\
\hline 31a & 37.28 & 30.51 & 21.43 & - & 141.54 & 114.17 \\
$31 \mathrm{~b}$ & 42.34 & 32.54 & 26.30 & 26.35 & 114.68 & 111.74 \\
\hline
\end{tabular}

Table 3. ${ }^{13} \mathrm{C}$ Chemical shifts of $\mathrm{C}[3]$ and $\mathrm{C}[5]$ in 32

\begin{tabular}{cccc}
\hline Compound & $\mathrm{X}$ & $\mathrm{C}[3], \mathrm{ppm}$ & $\mathrm{C}[5], \mathrm{ppm}$ \\
\hline 32a & $\mathrm{CH} 3$ & 30.97 & 22.64 \\
32b & $\mathrm{NH} 2$ & 31.51 & 21.37 \\
32c & $\mathrm{OH}$ & 31.73 & 20.60 \\
32d & $\mathrm{Cl}$ & 33.26 & 22.44 \\
32e & $\mathrm{Br}$ & 34.09 & 23.49 \\
32f & $\mathrm{I}$ & 34.70 & 24.74 \\
\hline
\end{tabular}


been widely used to determine the configuration of six - membered ring compounds. Thus, the $\beta$ - isomer of 1,3 - dimethyl -4 - phenylpiperidine ${ }^{68}$ has been assigned the cis configuration 33 with an axial orientation of the methyl group at C-3, based on the observation that C-5 resonates upfield [24.8 $\mathrm{ppm}]$ as compared with that in 1-methyl - 4 - phenylpiperidine[ 32.9 ppm ]

Jones et $\mathrm{al},{ }^{3}$ have assigned an axial oriental for the phenyl group in the $\alpha$ - isomer of 4 - hydroxyl - 1,2 dimethyl - 4 - phenylpiperidine[34a], based on the upfield shift of the ipso carbon [by 3-8 ppm] compared with that in the $\beta$ - isomer [34b] where the phenyl group is equatorial.

Jones and co - workers have assigned the configurations of methyl and phenyl groups in various isomeric 1, 2, 5 - trimethyl - 4 - phenylpiperidines, 4 - hydroxyl 1, 2, 5 - trimethyl - 4 - phenylpiperidines $^{70}$ and $4-$ hydroxyl - 1, 3, 5 - trimethyl - 4- phenylpiperidines ${ }^{71}$, using ${ }^{13} \mathrm{C}$ chemical shifts.

From a study of various di - and tri - methylcyclohexanes, Grant and dalling have found that an axial methyl group shifts the resonance of C[2], C[3] and C[4] by $1.40,5.41$ and $-6.37 \mathrm{ppm}$, whereas for an equatorial methyl group the corresponding shifts are 5.96, 9.03 and 0.05 respectively. The shielding by an axial methyl group, relative to an equatorial methyl group has been attributed to steric interactins.

Schneider and Hoppen ${ }^{4}$ have studied the $\alpha, \beta$ and $\gamma$ effects of various substituents $\mathrm{X}[\mathrm{X}=\mathrm{F}, \mathrm{Cl}, \mathrm{Br}, \mathrm{I}, \mathrm{OH}$, $\mathrm{OMe}]$ in $35 \mathrm{a}$ and $35 \mathrm{~b}$. It has been found that the magnitude and even the sign of substituent effects can be changed by introducing additional alkyl groups in the $\alpha, \beta$ and $\gamma$ positions. The shifts induced on $\beta$ carbons are found to be independent of the nature of the substituent $\mathrm{X}$.

The ${ }^{13} \mathrm{C}$ NMR data of 4 - hydroxypiperidines[36] have been analysed ${ }^{5}$ and compared with those of analogous piperidines. The results indicate that substituent effects are markedly influenced by steric interaction.

Table 4. One - bond

\begin{tabular}{lc}
\multicolumn{2}{c}{ coupling constants, ${ }^{1} \mathrm{~J}_{\mathrm{CH}}$} \\
\hline Methane & 125.0 \\
Ethane & 124.9 \\
Propane & 114.2 \\
Chloromethane & 150.0 \\
Methanol & 141.0 \\
Benzene & 165.0 \\
\hline
\end{tabular}

A method of assigning the configuration of a substituent in saturated six-mebered ring compounds existing in chair conformation, from ${ }^{13} \mathrm{C}$ chemical shift of a single epimer has been suggested by Pandiarajan and Manimekalai ${ }^{76}$.

\section{$2.2{ }^{13} \mathrm{C}-{ }^{1} \mathrm{H}$ Coupling Constants}

The ${ }^{13} \mathrm{C}-{ }^{1} \mathrm{H}$-coupling constants are useful parameters in the study of organic molecules.

One bond coupling constant ${ }^{1} \mathrm{~J}_{\mathrm{CH}}$ is given by equation 9 , where \%s represents the percentage ' $s$ ' character of the carbon hybrid orbital participating in the $\mathrm{C}-\mathrm{H}$ bond.

$$
{ }^{1} \mathrm{~J}_{\mathrm{CH}}=5 \mathrm{X}[\% \mathrm{~s}][\mathrm{Hz}]
$$

Equation [9] was later modified 79 as equation [10]

$$
{ }^{1} \mathrm{~J}_{\mathrm{CH}}=5.7 \mathrm{X}[\% \mathrm{~s}]-18.4[\mathrm{~Hz}]
$$

The ${ }^{1} \mathrm{~J}_{\mathrm{CH}}$ coupling constants in some compounds are given in Table 4.

${ }^{2} \mathrm{~J}_{\mathrm{CH}}$ coupling constants are usually small. The ${ }^{2} \mathrm{~J}_{\mathrm{CH}}$ coupling constants of some compounds are given in Table 5.

${ }^{3} \mathrm{~J}_{\mathrm{CH}}$ coupling constants depend on the dihedral angle. The coupling constant and dihedral angle are related by Karplus Conroy relation ( eqution 11$)^{6}$

$$
{ }^{3} \mathrm{~J}_{\mathrm{CH}}=4.26-\cos \varphi+3.56 \cos 2 \varphi
$$

Table 5. Two - bond

\begin{tabular}{cc}
\multicolumn{2}{c}{ coupling constants, ${ }^{2} \mathrm{~J}_{\mathrm{CH}}$} \\
\cline { 1 - 2 } Compound & ${ }^{1} \mathrm{~J}_{\mathrm{CH}[\mathrm{Hz}]}$ \\
\hline Ethane & -4.5 \\
Ethene & -2.4 \\
Ethyne & 49.3 \\
Benzene & 1.0 \\
Ethanol & 26.7 \\
\hline
\end{tabular}

Table 6. Three - bond coupling constants, ${ }^{3} \mathrm{~J}_{\mathrm{CH}}$

\begin{tabular}{cc}
\hline Compound & ${ }^{1} \mathbf{J}_{\mathrm{CH}[\mathrm{Hz}]}$ \\
\hline Pyridine & $\mathrm{C}[2]-\mathrm{H}[4]=6.9$ \\
& $\mathrm{C}[4]-\mathrm{H}[2]=6.3$ \\
\multirow{5}{*}{ Furan } & $\mathrm{C}[3]-\mathrm{H}[5]=6.6$ \\
& $\mathrm{C}[2]-\mathrm{H}[6]=11.2$ \\
& $\mathrm{C}[2]-\mathrm{H}[4]=7.0$ \\
& $\mathrm{C}[2]-\mathrm{H}[5]=6.9$ \\
& $\mathrm{C}[3]-\mathrm{H}[5]=6.0$ \\
\hline
\end{tabular}


${ }^{13} \mathrm{~J}_{\mathrm{CH}}$ coupling constants of some compounds are given in Table 6.

${ }^{13} \mathrm{C}-{ }^{13} \mathrm{C}$ coupling is usually not observed, except in compounds that have been deliberately enriched with ${ }^{13} \mathrm{C}$, because of the low probability of two adjacent ${ }^{13} \mathrm{C}$ atoms in a molecule.

${ }^{1} J_{c c}$ depends on the hybridisation of the two carbon atoms involved. In terms of the's' character of the two bonding hybrid orbitals, ${ }^{1} \mathrm{~J}_{\mathrm{cc}}$ may be given by equation 12.

$$
{ }^{1} \mathrm{~J}_{\mathrm{cx}} \mathrm{J}_{\mathrm{cy}}=7.3[\% \mathrm{sx}][\% \mathrm{sy}] / 100-17 \mathrm{~Hz}
$$

${ }^{1} \mathrm{~J}_{\mathrm{cc}}$ coupling constants of some compounds are given

The ${ }^{2} \mathrm{~J}_{c c}$ and ${ }^{3} \mathrm{~J}_{c c}$ coupling constants of some compounds are given in Table 8.

The values of ${ }^{1} \mathrm{~J}_{\mathrm{CH}},{ }^{2} \mathrm{~J}_{\mathrm{CH}},{ }^{3} \mathrm{~J}_{\mathrm{CH}},{ }^{1} \mathrm{~J}_{\mathrm{CC}},{ }^{2} \mathrm{~J}_{\mathrm{CC}},{ }^{3} \mathrm{~J}_{\mathrm{CC}}$ in various aliphatic, alicyclic, aromatic and heterocyclic compounds have been reported.

\subsection{Two - Dimensional NMR Spectroscopy}

In one - dimensional NMR spectrum, the abscissa corresponds to the frequency axis and ordinate gives the intensities of the signals. In a two dimensional NMR spectrum, both the abscissa and the ordinate are frequency axes, with the intensities constituting a third.

The two-dimensional methods are based on the coupling between nuclei. The most important two dimensional NMR spectra are those of the type which show either ${ }^{1} \mathrm{H}$ vs ${ }^{1} \mathrm{H}$ or ${ }^{1} \mathrm{H}$ vs ${ }^{13} \mathrm{C}$ chemical shift correlations.

Some of the important types of $2-\mathrm{D}$ experiments are given below:

\subsection{Homonuclear Correlation Spectroscopy [HOMOCOSY]}

In this type of spectrum, ${ }^{1} \mathrm{H}$ chemical shifts along both frequency axes are correlated with each other. The ${ }^{1} \mathrm{H}$ spectrum appears along the diagonal as contours responding

Table 7. One - bond coupling constants, ${ }^{1} \mathrm{~J}_{\mathrm{cc}}$

\begin{tabular}{lc}
\hline Compound & ${ }^{1} \mathrm{~J}_{c \mathrm{H}[\mathrm{Hz}]}$ \\
\hline Ethane & 34.6 \\
Benzene & 57.0 \\
Furan & 69.1 \\
Ethanol & 37.7 \\
\hline
\end{tabular}

peak intensities. The off - diagonal contours are the cross peaks. A horizontal line drawn from a cross peak will intercept a contour on the diagonal and a vertical line from the same cross peak will intercept another contour on the diagonal with which the first diagonal contour is coupled. Thus we can assign signals unambiguously.

The basic COSY gives the same information, obtained through the classical ${ }^{1} \mathrm{H}-{ }^{1} \mathrm{H}$ decoupling procedure. In general, strongly coupled protons are better handled a with COSY experiment than with conventional ${ }^{1} \mathrm{H}-{ }^{1} \mathrm{H}$ decoupling.

The basic COSY, however, often results in considerable overlap along the diagonal. Thus, it is difficult to make assignments. This can be alleviated by a double quantum filtered COSY [DQFCOSY].

For example, in the basic COSY spectrum of geraniol ${ }^{7}$ [37] (Table 7), the signals for the three methyl groups are severely overlapped, as are those for the two methyl groups. However, in the DQFCOSY spectrum (Table 8) $\mathrm{H}-8$ and $\mathrm{H}-9$ methyl proton signals are cleanly separated; and the small allylic coupling to $\mathrm{H}-6$ is apparent through the off - diagonal cross peak. The COSY lacks the $\mathrm{H}-1, \mathrm{H}-4$ and the $\mathrm{H}-2, \mathrm{H}-4$ couplings that are present in the DQFCOSY.

\subsection{Heteronuclear Correlation Spectroscopy [HETCOR]}

The HETCOR spectrum correlates the peaks of ${ }^{1} \mathrm{H}$ spectrum with the peaks of ${ }^{13} \mathrm{C}$ spectrum, and shows the specific protons attached to each carbon. The ${ }^{1} \mathrm{H}$ spectrum is presented on the vertical axis and the ${ }^{13} \mathrm{C}$ spectrum on the horizontal axis . The ${ }^{1} \mathrm{H}-{ }^{13} \mathrm{C}$ correlation is shown by the off - diagonal contours.

In a HETCOR spectrum, ${ }^{13} \mathrm{C}$ nuclei have much wider chemical shift ranges than protons, and are a collection of singlets, due to the absence of homonuclear coupling. Thus, the information about proton frequencies can be gained from the dispersed ${ }^{13} \mathrm{C}$ signals more accurately.
Table 8. Two - bond and three bond coupling constants, ${ }^{2} \mathrm{Jcc}_{\mathrm{cc}}$ and ${ }^{3} \mathrm{~J}_{\mathrm{cc}}$

\begin{tabular}{lcc}
\hline Compound & ${ }^{2} \mathrm{~J}_{\mathrm{cc}[\mathrm{Hz}]}$ & ${ }^{3} \mathrm{~J}_{\mathrm{cc}[\mathrm{Hz}]}$ \\
\hline Propyne & 11.8 & - \\
Cyclobutanone & 9.5 & - \\
Pyridine & - & 13.95 \\
Aniline & - & 7.9 \\
\hline
\end{tabular}


Evans et al. have used the HETCOR spectrum to assign the carbon signals of 38 , a natural product extracted from beans. Structural elucidation has been made for complicated molecules like 39 and 40.

\subsection{Nuclear Overhauser and Exchange Spectroscopy [NOESY]}

Nuclear Overhauser and Exchange Spectroscopy assembles all the ${ }^{1} \mathrm{H}-{ }^{1} \mathrm{H}$ nOe spectrum effects in a molecule into a single spectrum. In a NOESY spectrum, the ${ }^{1} \mathrm{H}$ spectrum is represented by contours along the diagonal, and the off diagonal contours represent non-bonding ${ }^{1} \mathrm{H}-{ }^{1} \mathrm{H}$ interactions with protons that are nearby in space.

Many attempts have been made to improve the practical techniques in $2-\mathrm{D}$ NMR and to make structural elucidation and conformational assignment of complicated molecules ${ }^{8}$.

\section{B - NMR Measurements}

Samples were prepared by dissolving about $10 \mathrm{mg}$ of the compounds in $0.5 \mathrm{ml}$ of DMSO $-\mathrm{d}_{6}$ containing $1 \%$ TMS for recording $1 \mathrm{H} \mathrm{NMR}$ and two dimensional NMR spectra. Samples were prepared by dissolving about $50 \mathrm{mg}$ of the compounds in $0.5 \mathrm{ml}$ of DMSO $-\mathrm{d}_{6}$ containing $1 \%$ TMS for recording ${ }^{13} \mathrm{CH}$ NMR. The spectral parameters are given below:

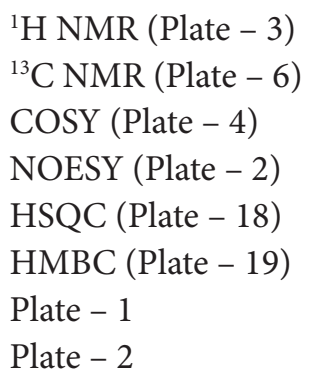

$$
\begin{aligned}
& \text { Plate - } 3 \\
& \text { Plate }-4 \\
& \text { Plate - } 5 \\
& \text { Plate - } 6 \\
& \text { Plate - } 7 \\
& \text { Plate - } 8 \\
& \text { Plate - } 9 \\
& \text { Plate - } 10 \\
& \text { Plate - 11 } \\
& \text { Plate - } 12 \\
& \text { Plate - } 13 \\
& \text { Plate - } 14 \\
& \text { Plate - } 15 \\
& \text { Plate - } 16 \\
& \text { Plate - } 17 \\
& \text { Plate - } 18 \\
& \text { Plate - } 19
\end{aligned}
$$

\section{References}

1. Thomas WA. NMR (Nuclear Magnetic Resonance) Spectroscopy. Annu Rev. 1968; 1:43.

2. Bladon P. NMR (Nuclear Magnetic Resonance) Spectroscopy. Annu Rev. 1969; 2:1.

3. Thomas WA. NMR (Nuclear Magnetic Resonance) Spectroscopy. Annu Rev. 1970; 3:91.

4. Anet FAL, Anet R. Determination of organic structure from physical methods. In Nachol FC, editor. New York: Academic Press; 1971.

5. Anet FAL, Anet R. Dynamic nuclear magnetic resonance spectroscopy. In Jackman LM, Cotton FA. New York: Academic Press; 1975.

6. Lukas G. Chem France. Bull Soc. 351; 1972.

7. Tanable M, Biosynthesis specialist periodical reports. In Geissman TA, editors. London: The Chemical Society; 1973.

8. Lambert JB, Featherman SI. Chem Rev. 1975; 75:611. 